\title{
Resonant States \\ of a Momentum-Dependent Impurity Potential in a $d$-Wave Superconductor
}

\author{
P. PisArski And G. HARAŃ \\ Institute of Physics, Politechnika Wrocławska \\ Wybrzeże Wyspiańskiego 27, 50-370 Wrocław, Poland
}

\begin{abstract}
Nonmagnetic impurity on-site potential leads to the resonant states in a $d$-wave superconductor for the unitary scattering limit. We extend the above study to include a momentum dependence of the impurity potential and discuss the existence and number of resonant states for a tetragonal anisotropy of the scattering potential.
\end{abstract}

PACS numbers: 74.20.Rp, 74.62.Dh, 74.72.-h

\section{Introduction}

Controlled impurity substitution is an important tool in the identification of a symmetry of the superconducting ground state. Particularly efficient are the scanning tunneling microscopy (STM) measurements of the local density of states (LDOS) which probe directly and with atomic precision excited states in the vicinity of the impurity atom. STM measurements of LDOS around single Zn [1] or Ni [2] impurity atom as well as $\mathrm{Cu}$ vacancy [3] in superconducting $\mathrm{Bi}_{2} \mathrm{Sr}_{2} \mathrm{CaCu}_{2} \mathrm{O}_{8+\delta}$ revealed a distinct fourfold symmetry of LDOS specific for a $d$-wave superconductor. Although, the main features of complex STM patterns in cuprates are captured by a model on-site impurity scattering [4-6], studies of extended impurity potentials [7-9] are motivated by some still unresolved features of LDOS in these compounds [10]. We continue the issue of anisotropic in the reciprocal space impurity potential [11-15] and discuss its effect on the impurity-bound states in high-temperature superconductors.

\section{Momentum-dependent impurity potential}

We consider quasiparticle scattering in a $d$-wave superconducting state by a momentum-dependent nonmagnetic impurity potential 


$$
\hat{v}\left(\boldsymbol{k}, \boldsymbol{k}^{\prime}\right)=\left[v_{\mathrm{i}}+v_{\mathrm{a}} f(\boldsymbol{k}) f\left(\boldsymbol{k}^{\prime}\right)\right] \hat{\tau}_{3},
$$

where $v_{\mathrm{i}}, v_{\mathrm{a}}$ represent the isotropic and anisotropic amplitudes of the potential, respectively. We rewrite $v_{\mathrm{i}}$ and $v_{\mathrm{a}}$ in terms of an overall impurity potential $v$ and a potential partition parameter $\alpha(0 \leq \alpha \leq 1)$ which divides $v$ into isotropic and anisotropic channels: $v_{\mathrm{i}}=\alpha v, v_{\mathrm{a}}=(1-\alpha) v$. The symmetry of the potential is determined by the momentum-dependent function $f(\boldsymbol{k})$, which vanishes when integrated over the Fermi surface (FS) momenta $\langle f\rangle=\int_{\mathrm{FS}} \mathrm{d} S_{k} n(\boldsymbol{k}) f(\boldsymbol{k})=0$, where $n(\boldsymbol{k})$ is a normalized angle resolved FS density of states, $\int_{\mathrm{FS}} \mathrm{d} S_{k} n(\boldsymbol{k})=1$. $\hat{\tau}_{0}$ is the identity and $\hat{\tau}_{i}(i=1,2,3)$ are the Pauli matrices in the particle-hole space. We are particularly interested in a tetragonal anisotropy of the impurity potential, which may correspond to the potential of the scattering center in the high-temperature superconductor. Therefore, we study the case of the anisotropy functions $f(\boldsymbol{k})=\operatorname{sgn}(\cos 2 l \varphi)$ and $f(\boldsymbol{k})=\operatorname{sgn}(\sin 2 l \varphi)$, where $l$ is an integer number, which are the basis functions of the $C_{4 v}$ one-dimensional irreducible representations.

\section{Local density of states}

We discuss the effect of the impurity scattering potential (1) on the quasiparticle resonant states by analyzing the local density of states at zero temperature

$$
N(\boldsymbol{r}, \omega)=-\frac{1}{\pi} \operatorname{Im}\left[\sum_{\boldsymbol{k}} \hat{G}_{0}(\boldsymbol{k}, \omega)+\delta \hat{G}(\boldsymbol{r}, \boldsymbol{r}, \omega)\right]_{11},
$$

which is given by the diagonal element of the retarded Green function of a pure superconducting system $\hat{G}_{0}(\boldsymbol{k}, \omega)$ modified by the impurity-induced change of the spatial Green function $\delta \hat{G}(\boldsymbol{r}, \boldsymbol{r}, \omega)$. The Green function of a uniform superconductor reads

$$
\hat{G}_{0}(\boldsymbol{k}, \omega)=\left(\omega \hat{\tau}_{0}-\xi_{\boldsymbol{k}} \hat{\tau}_{3}-\Delta_{\boldsymbol{k}} \hat{\tau}_{1}\right)^{-1},
$$

where $\xi_{\boldsymbol{k}}$ is the quasiparticle energy in the normal state and $\Delta_{\boldsymbol{k}}$ is the superconducting order parameter. The position-dependent part of the Green function is determined in a simplified T-matrix approach appropriate for a single impurity effect

$$
\delta \hat{G}(\boldsymbol{r}, \boldsymbol{r}, \omega)=\sum_{\boldsymbol{k}, \boldsymbol{k}^{\prime}} \mathrm{e}^{\mathrm{i}\left(\boldsymbol{k}-\boldsymbol{k}^{\prime}\right) \boldsymbol{r}} \hat{G}_{0}(\boldsymbol{k}, \omega) \hat{T}\left(\boldsymbol{k}, \boldsymbol{k}^{\prime}, \omega\right) \hat{G}_{0}\left(\boldsymbol{k}^{\prime}, \omega\right),
$$

where the T-matrix is given by a self-consistent equation

$$
\hat{T}\left(\boldsymbol{k}, \boldsymbol{k}^{\prime}, \omega\right)=\hat{v}\left(\boldsymbol{k}, \boldsymbol{k}^{\prime}\right)+\sum_{\boldsymbol{k}^{\prime \prime}} \hat{v}\left(\boldsymbol{k}, \boldsymbol{k}^{\prime \prime}\right) \hat{G}_{0}\left(\boldsymbol{k}^{\prime \prime}, \omega\right) \hat{T}\left(\boldsymbol{k}^{\prime \prime}, \boldsymbol{k}^{\prime}, \omega\right) .
$$

Due to the separable form of the impurity potential (1) the T-matrix factorizes into four matrix components $\hat{T}_{i}(\omega)[12]$ :

$$
\hat{T}\left(\boldsymbol{k}, \boldsymbol{k}^{\prime}, \omega\right)=\hat{T}_{0}(\omega)+\hat{T}_{1}(\omega) f(\boldsymbol{k}) f\left(\boldsymbol{k}^{\prime}\right)+\hat{T}_{2}(\omega) f(\boldsymbol{k})+\hat{T}_{3}(\omega) f\left(\boldsymbol{k}^{\prime}\right)
$$

and yields a simple formula for the local density of states 


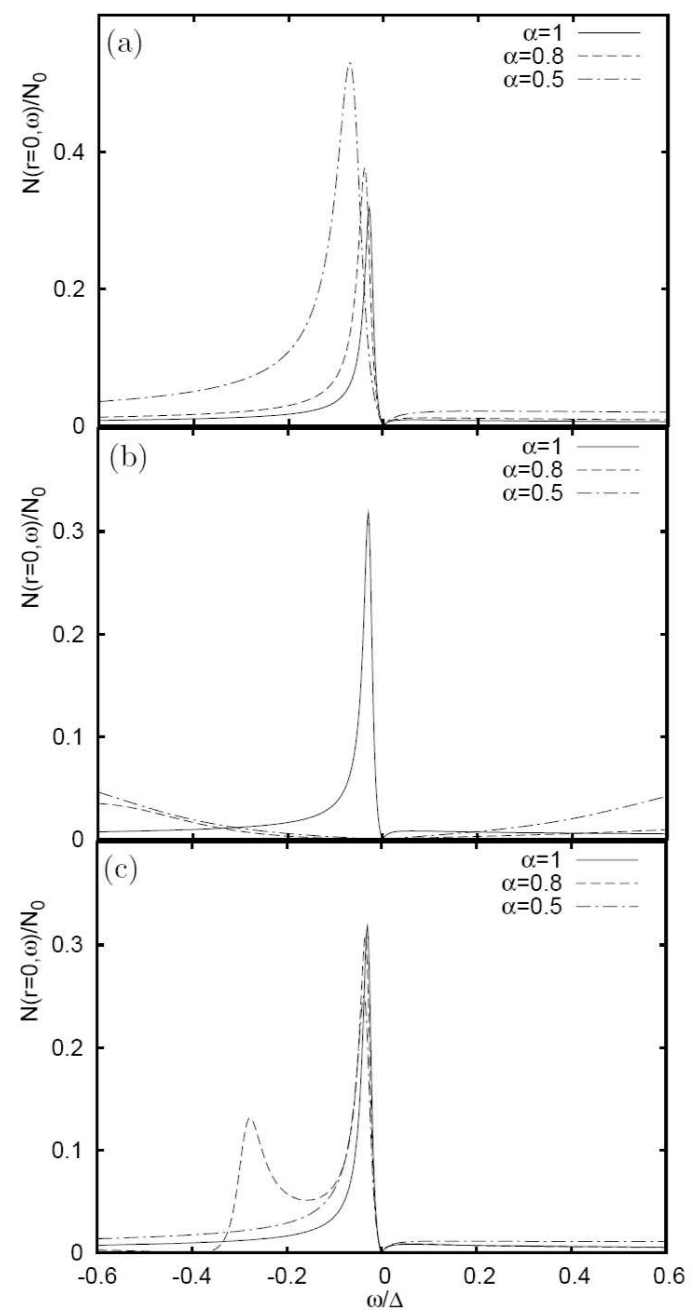

Fig. 1. Relative density of states $N(\boldsymbol{r}, \omega) / N_{0}$ at the impurity site $\boldsymbol{r}=0$ for isotropic (solid line) and anisotropic scattering determined by $f(\boldsymbol{k})=\operatorname{sgn}(\sin 2 l \varphi)$ (a), anisotropic scattering $f(\boldsymbol{k})=\operatorname{sgn}(\cos 2 \varphi)(\mathrm{b})$, and $f(\boldsymbol{k})=\operatorname{sgn}(\cos 4 \varphi)(\mathrm{c}) . N_{0}=m / 2 \pi$ is a single spin normal-state density of states at the Fermi energy.

$$
\begin{aligned}
& N(\boldsymbol{r}, \omega)=-\frac{1}{\pi} \operatorname{Im}\left[\hat{G}_{0}(\boldsymbol{r}=0, \omega)\right. \\
& \quad+\hat{G}_{0}(\boldsymbol{r}, \omega) \hat{T}_{0}(\omega) \hat{G}_{0}(-\boldsymbol{r}, \omega)+\hat{G}_{1}(\boldsymbol{r}, \omega) \hat{T}_{1}(\omega) \hat{G}_{1}(-\boldsymbol{r}, \omega) \\
& \left.\quad+\hat{G}_{1}(\boldsymbol{r}, \omega) \hat{T}_{2}(\omega) \hat{G}_{0}(-\boldsymbol{r}, \omega)+\hat{G}_{0}(\boldsymbol{r}, \omega) \hat{T}_{3}(\omega) \hat{G}_{1}(-\boldsymbol{r}, \omega)\right]_{11}
\end{aligned}
$$

where

$$
\hat{G}_{j}(\boldsymbol{r}, \omega)=\sum_{\boldsymbol{k}} \mathrm{e}^{\mathrm{i} \boldsymbol{k} \boldsymbol{r}} f^{j}(\boldsymbol{k}) \hat{G}_{0}(\boldsymbol{k}, \omega), \quad j=0,1 .
$$


$\hat{G}_{0}(\boldsymbol{r}, \omega)$ is a two-dimensional Fourier transform of the uniform system Green function (3) and $\hat{G}_{1}(\boldsymbol{r}, \omega)$ includes the anisotropy of the potential. The quasiparticle excitation energy spectrum is determined by the poles of the Green function (4), which coincide with the poles of the spatial Green function of a uniform system $\hat{G}_{0}(\boldsymbol{r}, \omega)$, and the poles of the scattering matrix $\hat{T}_{i}(\omega)$. Only the poles of the T-matrix depend on the impurity potential and therefore determine the impurity states. We discuss the existence, number and energy of resonant and virtual resonant (finite quasiparticle lifetime) states at the impurity site $(r=0)$. In the calculations we take a parabolic quasiparticle dispersion in a normal state, $\xi_{\boldsymbol{k}}=k^{2} / 2 m-\varepsilon_{\mathrm{F}}$, where $\varepsilon_{\mathrm{F}}$ is the Fermi energy, and the $d$-wave superconductivity $\Delta_{k}=\Delta\left(k_{x}^{2}-k_{y}^{2}\right)$. Assumption of a parabolic quasiparticle dispersion, acceptable for overdoped cuprates, allows us to concentrate on a feature of coupled order parameter and impurity potential anisotropies. We also put $\hbar=1$. Our results are summarized in Fig. 1.

\section{Results}

We have evaluated LDOS at the impurity site for various potentials (1) of a tetragonal symmetry, which according to their effect on the resonant states can be classified into three groups defined by the anisotropy function $f(\boldsymbol{k})=\operatorname{sgn}(\sin 2 l \varphi)$, $\operatorname{sgn}(\cos 4 l \varphi)$, and $\operatorname{sgn}(\cos 2(2 l+1) \varphi)$. The impurity potential given by $f(\boldsymbol{k})=$ $\operatorname{sgn}(\sin 2 l \varphi)$ has no effect on the LDOS at the impurity site compared to the case of isotropic scattering. The elements $\hat{T}_{2}$ and $\hat{T}_{3}$ of the scattering matrix are equal to zero and $\hat{T}_{1}$ is multiplied by $\hat{G}_{1}(\boldsymbol{r}, \omega)$ which for $\boldsymbol{r}=0$ is also equal to zero. The only nonvanishing element of the Green function $\delta \hat{G}(\boldsymbol{r}, \boldsymbol{r}, \omega)$ is the one with $\hat{T}_{0}$, just like in the case of isotropic impurity scattering (Fig. 1a). Therefore, the resonant state appears at the Fermi energy only in the limit of unitary scattering $[4,5]$. Scattering by the potential determined by $f(\boldsymbol{k})=\operatorname{sgn}(\cos 2(2 l+1) \varphi)$ smears out the density of states around the impurity and eliminates the resonant states, which we present in Fig. $1 \mathrm{~b}$ for $l=0$. The most interesting effect is found for $f(\boldsymbol{k})=\operatorname{sgn}(\cos 4 l \varphi)$, shown for $l=1$ in Fig. 1c. Except for the equal partition of the impurity potential into isotropic and anisotropic channels $(\alpha=0.5)$ we observe two virtual resonant states which merge and become a resonant state in the limit of unitary scattering.

\section{Conclusions}

Summarizing, we have found that the momentum-dependent scattering potential modifies the impurity quasiparticle states. A tetragonal symmetry of the impurity potential, possible in cuprates, can induce three different effects in the local density of states around a scattering center. It can lead to a development of a pair of virtual resonant states, preserve the impurity bound states generated by the isotropic scattering potential, or destroy these states. 


\section{References}

[1] S.H. Pan, E.W. Hudson, K.M. Lang, H. Eisaki, S. Uchida, J.C. Davis, Nature 403, 746 (2000).

[2] E.W. Hudson, K.M. Lang, V. Madhavan, S.H. Pan, H. Eisaki, S. Uchida, J.C. Davis, Nature 411, 920 (2001).

[3] E.W. Hudson, V. Madhavan, K. McElroy, J.E. Hoffmann, K.M. Lang, H. Eisaki, S. Uchida, J.C. Davis, Physica B 329, 1365 (2003).

[4] A.V. Balatsky, M.I. Salkola, A. Rosengren, Phys. Rev. B 51, 15547 (1995).

[5] M.I. Salkola, A.V. Balatsky, S.J. Scalapino, Phys. Rev. Lett. 77, 1841 (1996).

[6] A.V. Balatsky, I. Vekhter, J.X. Zhu, Rev. Mod. Phys. 78, 373 (2006).

[7] A.P. Kampf, T.P. Devereaux, Phys. Rev. B 56, 2360 (1997).

[8] J.M. Tang, M.E. Flatté, Phys. Rev. B 70, 140510 (2004).

[9] C.H. Choi, Phys. Rev. B 63, 064507 (2001).

[10] A.V. Balatsky, I. Vekhter, Jian-Xin Zhu, Rev. Mod. Phys. 78, 373 (2006).

[11] G. Harań, A.D.S. Nagi, Phys. Rev. B 54, 15463 (1996).

[12] G. Harań, A.D.S. Nagi, Phys. Rev. B 58, 12441 (1998).

[13] P. Pisarski, G. Harań, Physica C 390, 270 (2003).

[14] A. Maciag, P. Pisarski, G. Harań, Acta Phys. Pol. B 34, 479 (2003).

[15] A. Maciag, P. Pisarski, G. Harań, Physica C 387, 73 (2003). 